\title{
Development and evaluation of a new contoured cushion system with an optimized normalization algorithm
}

\author{
Sujiao $\mathrm{Li}^{\mathrm{a}}$, Zhengxiang Zhang ${ }^{\mathrm{b}}$ and Jue Wang ${ }^{\mathrm{a},{ }^{*}}$ \\ ${ }^{a}$ The Key Laboratory of Biomedical Information Engineering of Ministry of Education, and Institute of \\ Biomedical Engineering, School of Life Science and Technology, Xi'an Jiaotong University, Shaanxi, \\ 710065, P.R. China \\ ${ }^{b}$ Shaanxi Bo'ai Hospital, Xi'an, Shaanxi, 710065, P.R. China
}

\begin{abstract}
Prevention of pressure sores remains a significant problem confronting spinal cord injury patients and the elderly with limited mobility. One vital aspect of this subject concerns the development of cushions to decrease pressure ulcers for seated patients, particularly those bound by wheelchairs. Here, we present a novel cushion system that employs interface pressure distribution between the cushion and the buttocks to design custom contoured foam cushion. An optimized normalization algorithm was proposed, with which interface pressure distribution was transformed into the carving depth of foam cushions according to the biomechanical characteristics of the foam. The shape and pressure-relief performance of the custom contoured foam cushions was investigated. The outcomes showed that the contoured shape of personalized cushion matched the buttock contour very well. Moreover, the custom contoured cushion could alleviate pressure under buttocks and increase subjective comfort and stability significantly. Furthermore, the fabricating method not only decreased the unit production cost but also simplified the procedure for manufacturing. All in all, this prototype seat cushion would be an effective and economical way to prevent pressure ulcers.
\end{abstract}

Keywords: Prototype, manufacture system, custom-contoured cushion (CCC), pressure sores, optimized normalization algorithm

\section{Introduction}

Pressure sores (known as pressure ulcers, bed sores and decubitus injuries) are localized areas of tissue necrosis believed to be caused by long-term high pressure, lack of blood supply, and impaired lymphatic drainage [1,2]. A few studies have pointed out that interface pressure, shear pressure, friction, and moisture are the four major factors contributing to the development of pressure sores [35]. Likewise, wheelchair-bound individuals with disability, the elderly, and prolonged sitting individuals are all at high risk of developing pressure sores [6-8]. It was reported by the Second National Sample Survey on Disability Office that, in China, there are 24.12 million individuals with

\footnotetext{
${ }^{*}$ Corresponding author: Jue Wang, The Key Laboratory of Biomedical Information Engineering of Ministry of Education, and Institute of Biomedical Engineering, School of Life Science and Technology, Xi'an Jiaotong University, Shaanxi, 710065, P.R. China. Tel.: +86 29 82663497; Fax: +86 29c82663497; E-mail: juewang1@126.com.
}

0959-2989/14/\$27.50 @ 2014 - IOS Press and the authors. 
extremity disability, accounting for $29.09 \%$ of all disabled individuals, and 144 million people over the age of $60,10 \%$ of them needing nursing care such as wheelchairs [9]. Currently, cushions are clinically prescribed to relieve, redistribute or reduce pressure in order to prevent pressure sores. Therefore, seat cushions with high quality and reasonable price is of particular importance in clinical rehabilitation and would be in huge demand for individuals suffering from pressure sores.

To date, several types of ulcers-preventing cushions have been described. One type of cushion filled with rubber, gel, or fluid materials could provide good performance for pressure relief [10-13]. However, this type of cushion is very weak for providing adequate support and is relatively expensive. Alternatively, polyurethane foam cushion with visco-elastic and resilient properties could provide sufficient support to maintain the buttock's shape, thereby providing sufficient dynamic stability [14]. Therefore, the foam cushion provides comparable performance in pressure relief at lower cost compared to fluid-filled cushion, it also offers the advantage of air permeability, flame retardance and low weight [3,14-19]. Moreover, some studies found that polyurethane foam produced lower peak interface pressure and offered better pressure distribution than gel did [20,21].

Current studies are directed at determining the shape of the load cushion rather than the method of its production. There were only few researchers who had developed specialized computer assistive design and manufacture (CAD/CAM) system for custom foam cushions and body supports. Brienza et al. $[3,14]$ designed the CAM system for custom-contoured foam cushion. This system measured the interface pressure loaded by support surface shape adjustment, then through electronic shape sensor to generate the seat contour. Yang and his colleagues [19] presented a prototype design and manufacture system that developed the custom-contoured cushions with interface pressure. They came up with the strategy that converted the interface pressure into corresponding milling depth of cushion according to its force-deflection characteristics. Silva $F$ et al. [22] proposed a customised seat with acquiring the user's contour through plaster cast. They scanned and manipulated the 3D cast to collect the surface geometry.

In this study, a new method for fabricating custom-contoured cushions is presented. Our aim is to design custom-contoured foam cushions based on interface pressure distribution and biomechanical properties of human buttocks. We came up with a new optimized normalization algorithm to transform interface pressure distribution into depth of milling foam cushions depending on load-deformation characteristics of the foam. This approach normalized the peak carving depth of foam cushion and avoided the "bottom out" of foam cushion under the ischial tuberosities [23]. This method simplified the manufacturing procedure and offers decreased production coast of CCC. Interface seat-pressure distribution and objective comfort were employed to compare foam-made CCCs and flat foam cushions for SCI and healthy prolonged sitting participants. Our results demonstrated that the CCC could redistribute interface pressure for prevent pressure sores while improving the overall comfort.

\section{Methods}

\subsection{System design}

This system is comprised of four portions: interface pressure measuring instruments (Tekscan, Boston MA, USA), design and manufacture platform of custom-contoured cushion, cutting machine (Easycut, Many 2022, Taiwan) and shape and performance evaluation platform of custom-contoured cushion. The procedure of the cushion design and its performance validation are displayed in Figure 1. The fabricating system is a four-degree of freedom cutting machine specially designed for foam 
cushions with viscoelastic and high-resiliency polyurethane properties, with which $\mathrm{x}, \mathrm{y}, \mathrm{z}$ axes displacements and Z-axis rotation were adjusted conveniently. We employed Tekscan system, CCC design and manufacture platform to design, fabricate, and evaluate the CCC. The Tekscan system is an interface pressure collecting instrument comprised of $42 \times 48$ array of pressure sensors. The cushion's surface shape and objective parameters of evaluation can be calculated from each pressure profile of the buttock.

$(A-1)$
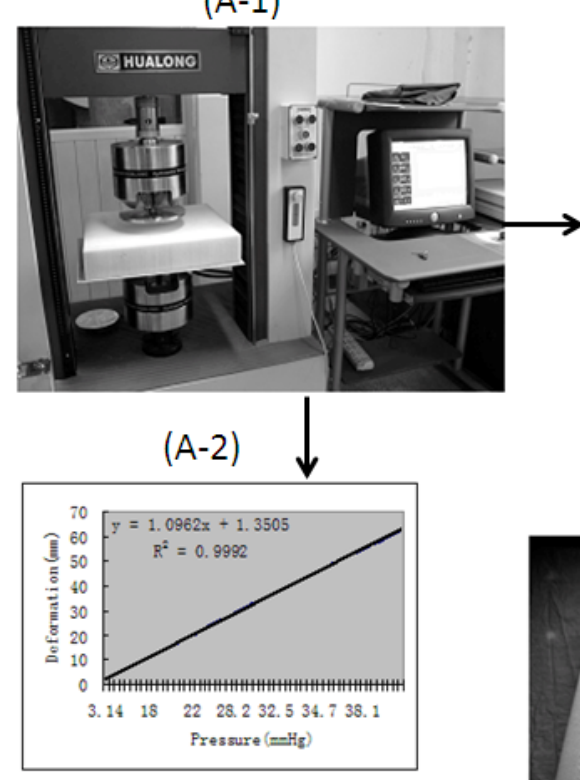

(B)

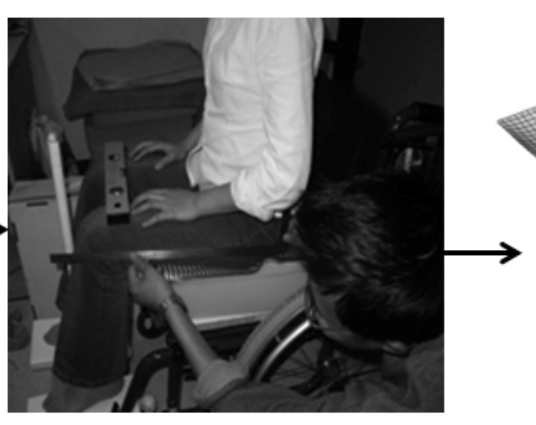

(E)

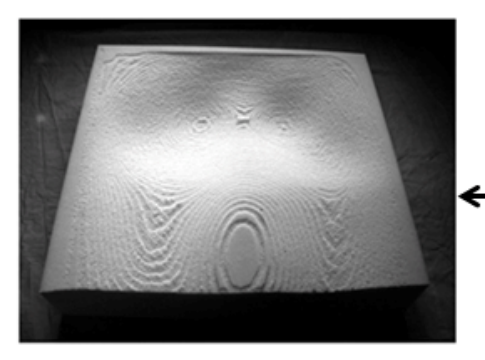

(C)

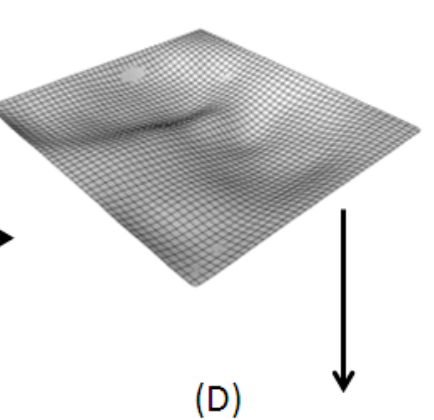

(D)

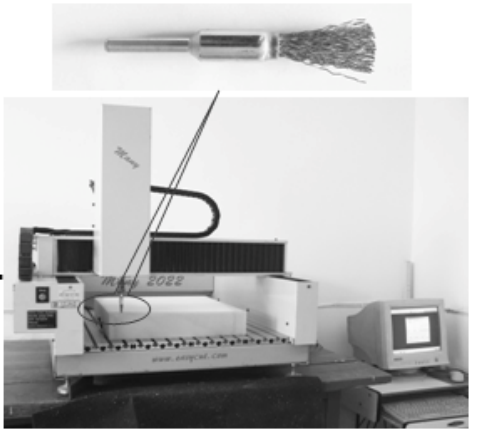

Fig. 1. Cushion design flowchart. (A-1) and (A-2) displayed the pressure-deformation testing system and the loaddeformation curve fitting function, respectively, here R2 was the correlation coefficient between pressure and foam deflection; (B) gave standard sitting posture to collect raw pressure data; (C) showed the surface contour for fabricating custom contoured cushion; (D) was the cutting configuration fabricating custom-contoured cushion, the cutting tools to manufacture foam cushion showed in the upper picture; (E) displayed the sample of custom contoured cushion.

\subsection{Cushion manufacture}

\subsubsection{Load-deformation test for foam}

Mechanical experiment program was carried out with WDW-100D mechanical testing machine, which was designed according to the American Standard Test Methods for Flexible Cellular Materials (ASTM D3574-95). The system is shown in Figure 1(A-1). This load-deformation test system included cylindrical steel indenter with $3.23 \times 10^{5} \mathrm{~mm}^{2}$ area and one hard aluminum board. There were some $6 \mathrm{~mm}$ diameter holes with $20 \mathrm{~mm}$ hole pitch on the aluminum board. The foam sample was the same as flat foam cushion with dimensions of 380-length, 420 -width, and 80 -height $\mathrm{mm}^{3}$.

The supporting base under aluminum board should be adjusted to the level. The foam cushion was put on the hard aluminum board and the center of cushion coincided with that of the indenter. After 
declining the indenter to touch foam cushion, we descended the indenter $1 \mathrm{~mm}$ by $1 \mathrm{~mm}$ with 60 -second pause and recorded the displacement of indenter and the pressure on the foam. Since foam cushion is viscoelastic and large deformation material, the linear relation of deformation and pressure would not keep with the deformation increasing until the indentor was more than $60 \mathrm{~mm}$ according to our experiments. Functional relation of pressure and deformation was obtained from fitting curve of the displacement and pressure value. As it is shown in Figure 1(A-2), the load-deflection functional relation was

$$
\mathrm{y}=1.0962 \mathrm{x}+1.3505
$$

Here, $\mathrm{y}$ is less than $60 \mathrm{~mm}$.

\subsubsection{Collecting interface pressure data}

The protocol of collecting interface pressure is as follows: a suitable size Hardboard was placed on a wheelchair, which would not distort when a $100 \mathrm{Kg}$ subject sits on it. Flat foam cushion used to manufacture CCC was put on the board, and Tekscan pressure sensors was placed on top of them, displayed in Figure 1(B). The interface pressures between flat cushion (FC) and buttocks were collected after calibrating the Tekscan system. MATLAB program transformed raw interface pressure matrix into the milling depth of CCC.

\subsubsection{The cushion model building with normalized method}

The interface pressure distribution $\mathbf{P}$ between buttock and flat foam cushion was recorded with Tekscan system, which is described by Eq. (2),

$$
\mathbf{P}=\left(\begin{array}{ccc}
p_{1,1} & \cdots & p_{1, C} \\
\cdots & \cdots & \cdots \\
p_{R, 1} & \cdots & p_{R, C}
\end{array}\right)
$$

Where $\mathrm{R}$ is 42 , the row size of force sensory array, and $\mathrm{C}$ is 48 , the column size of force sensory array.

The load versus deflection function derived from load-deformation test for foam experiment is shown in Figure 1 (A-2). According to this function, pressure distribution $\mathrm{P}$ can be converted into the corresponding depth matrix D based on one-to-one correspondence criteria. The depth deformation matrix is shown in Eq. (3),

$$
\mathbf{D}=1.0962 \times \mathbf{P}+1.3505
$$

Where $\mathrm{D}$ is deformation in $\mathrm{mm}, \mathrm{P}$ is pressure matrix in $\mathrm{mmHg}$.

In the process of design, we found that the maximum value of $\mathrm{D}$ sometimes came close to or exceeded the cushion's thickness, which would result in a non-linear load-deformation relationship with increasing deformation. In fact, if a cushion is too soft or too thin, it would "bottom out" [23], resulting in peak pressure increasing sharply. Therefore, a tradeoff between envelopment and "bottom 
out" should be taken into account. We pre-setted the maximum milling depth for certain cushion thickness to prevent "bottom out". The deflection coefficient was defined as the expected maximum milling depth divided by the maximum value in matrix $\mathrm{D}$, named as $\mathrm{K}$,

$$
\mathrm{K}=\operatorname{Dexp} / \max (1.0962 \times \mathrm{P}+1.3505)
$$

Here Dexp is the expected maximum milling depth for cushion, which is determined experimentally. Therefore, the real cutting matrix is

$$
\mathbf{D}_{\text {cutting }}=K(1.0962 \times P+1.3505)=\left(\begin{array}{ccc}
\mathrm{D}_{1,1} & \ldots & \mathrm{D}_{1,48} \\
\ldots & \ldots & \ldots \\
\mathrm{D}_{42,1} & \ldots & \mathrm{D}_{42,48}
\end{array}\right)
$$

For acquiring the optimized coefficient $K_{\text {opt }}$, we investigated the pressure-relieving performance of different carving peak values. The results demonstrated that the deepest carving deflection of $50 \mathrm{~mm}$ resulted in the best the performance at preventing pressure sores.

\subsubsection{CAD NURBS surface modeling}

To take into account that $\mathbf{D}_{\text {cutting }}$ sometimes contained many discontinuous fragments, which would exist difference from the final shape of cushion surface. Here the surface model of cushion was built by Rhinoceros NURBS modeling for windows, which converted the discontinuous fragments into NURBS surface of CCC, and decreased the noise significantly. The NURBS surface of CCC was saved into IGES, then the ASCII NC codes was converted by standard Mastercam software. The Figure 1(C) displayed the modeling surface of CCC.

Table 1

Anthropometry descriptive statistics

\begin{tabular}{lllll}
\hline Class & Age $($ years $)$ & Height $(\mathrm{m})$ & Weight $(\mathrm{Kg})$ & BMI $\left(\mathrm{Kg} / \mathrm{m}^{2}\right)$ \\
\hline Healthy subject $(\mathrm{n}=16)$ & $35.23 \pm 6.91(25-55)$ & $1.73 \pm 0.56(1.58-1.80)$ & $66.52 \pm 10.30(45-85)$ & $22.98 \pm 2.81(17.9-27.4)$ \\
SCI subjects $(\mathrm{n}=16)$ & $38.32 \pm 5.79(28-55)$ & $1.70 \pm 0.24(1.60-1.80)$ & $62.72 \pm 11.27(45-83)$ & $21.38 \pm 3.48(17.42-28.26)$ \\
\hline
\end{tabular}

Note: Mean \pm SD (Min-Max) (SD, standard deviation)

The modeling surface of CCC could be fabricated with our Easycut milling machine, shown in Figure 1(D). The cutting tool was a specialized design for cutting high-resiliency polyurethane foam and viscoelastic foam cushions efficiently and smoothly. Easycut2001 machine read the ASCII NC codes about carving depth and cut foam to CCC. The CCC is shown in Figure 1(E). 


\subsection{Custom-contoured evaluation protocol}

\subsubsection{Subjects}

Thirty-two participants with a high risk occurrence of pressure ulcers were recruited, they were classified into two groups: one group included sixteen SCI subjects and the other included sixteen prolonged sitting healthy (eight-hour of sitting per day) subjects. We enrolled SCI volunteers with spinal cord injury (T7-L2) coming from the rehabilitation center of the hospital, as well as agematched healthy subjects made up of students and teachers at the university. All subjects reported no pressure ulcers and neuromuscular disorders for at least one year. They were informed of the detailed procedure of whole study and signed the consent. This consent was approved by the Medical Ethics Committee.

Descriptive statistics on demographics of all participants such as age, height weight, and body mass index (BMI) are shown in Table 1. IP meant injured position of SCI participants, Valid N referred to the number of participants, and (BMI was computed by dividing the participant's weight in kilogram by their height in square meter.

\subsubsection{Foam materials selection for cushion}

The foams with different hardness from $25 \mathrm{~N}$ to $45 \mathrm{~N}$, on basis of 25\% IFD (indentation force deflection), were investigated as flat cushions. Outcomes displayed that the foam with $35 \mathrm{~N}$ hardness has the best performance in decreasing interface pressures. In addition, the foam itself has desirable characteristics such as air permeability, flame retardance (reaching the standard of GB/T 2408-1996 UL-94 HB), high strength (1 kg per square centimeter), resilience (double extensibility ratio), clinical security, and a density of $30 \mathrm{~kg}$ per cubic meter.

\subsubsection{Clinical evaluation}

All the subjects gave their subjective evaluations, and objective evaluations were recorded with standard sitting posture, namely, sitting on their custom-contoured cushions in wheelchair with centering pelvises medial laterally, trying to level pelvis and keep trunk upright, paralleling thighs to the level ground, relaxing hands on the laps, and facing straight forward. In order to decrease the errors caused by creep characteristics, patients stayed in wheelchair for five minutes before interface pressures were collected. Backrests and footrests were adjusted to make sure that participant had a relaxing seating-posture.

\subsubsection{Subjective evaluation}

The lateral stability (LS), anteroposterior stability (APS), and comfort degree (CD) [17] were employed in the procedure of subjective evaluation, which mainly represented the stability and comfort of the flat cushion and CCCs. All the subjects gave their opinions about FC and CCC on LS, APS and CD.

\subsubsection{Objective evaluation}

By keeping standard sitting posture, interface pressure arrays were measured using Tekscan sensor for each subject with both the flat foam cushion and CCC. If the pressure elements of data recorded a zero value, which stood for these locations where support elements without contacting the test subjects. Hence, these data were removed from analysis. The objective assessment parameters were calculated from the remaining data. The parameters calculated to assess pressure distribution were maximum 
pressure (MP) in Eq. (6), average pressure (AP) in Eq. (7), average pressure gradient (APG) in Eq. (8), and balance coefficient (BC) in Eq. (9).

$$
\begin{aligned}
& M P=\max (P) \\
& A P=\frac{1}{N p} \sum_{i=1}^{N p} P i
\end{aligned}
$$

Pressure gradient was the ratio of pressure change along certain direction, APG is,

$$
A P G=\frac{1}{N p} \sum_{i=0}^{N p}(\operatorname{grad} G i)
$$

$\mathrm{Np}$ was number of valid points bearing pressure, and gradGi was matrix of pressure gradient.

$$
B C=\frac{\left|T F P_{y}-R-T F P_{y}-L\right|}{\left|T F P_{y}-R+T F P_{y}-L\right|} \times 100
$$

Where TFPy $-\mathrm{R}$ and TFPy $-\mathrm{L}$ are the peak of right and left points in anterior-posterior TFPy (topfive project), respectively. TFPy is a vertical pressure vector of the mean of vertical largest, nextlargest,..., the $5^{\text {th }}$ largest pixel values of pressure distribution matrix. The small BC value demonstrates good balance performance for the cushion. If the SCI patients had exceptionally large $\mathrm{BC}$, medical staff or experimenter assisted the SCI patients in maintaining correct posture [24].

\subsection{Data analysis}

Two sets of objective and subjective pressure data were generated with FC and CCC. The objective and subjective data were divided in to two groups: the healthy and SCI groups. Paired-sample t-test was performed on the evaluation parameters to analyze the two sets of objective and subjective pressure parameters from the healthy and SCI groups to see whether significant differences existed between FC and CCC.

\section{Results}

\subsection{Validation of the shape of custom contoured foam cushion}

To verify the custom accuracy of foam cushion, The CCC from a typical subject was investigated to determine the differences of the contour of buttock shape carved into foam cushion and the carving depth of the contoured cushion. The foam cushion's upper level surface was used as a reference plane. 
We selected two-side maximum points under the ischial tuberosity and four points were chosen around each of peak point. Two points in two trunks joined in the point list. Total of twelve sample points' depth on carved foam cushion were measured. Then marking the roughly corresponding locations of

Table 2

Differences evaluation between the buttock shape and contoured cushion

\begin{tabular}{llll}
\hline Location N.O & Carved depth $(\mathrm{mm})$ & Protruding depth $(\mathrm{mm})$ & Absolute error ${ }^{\#}(\mathrm{~mm})$ \\
\hline 1 & 49.8 & 52.2 & 2.4 \\
2 & 27.0 & 27.8 & 0.8 \\
3 & 30.5 & 32.1 & 1.6 \\
4 & 28.1 & 29.4 & 1.3 \\
5 & 27.9 & 28.3 & 1.4 \\
6 & 19.4 & 20.1 & 0.7 \\
7 & 49.4 & 51.5 & 2.1 \\
8 & 31.1 & 32.5 & 1.4 \\
9 & 27.9 & 28.3 & 0.8 \\
10 & 33.0 & 32.6 & -0.5 \\
11 & 31.2 & 33.4 & 2.2 \\
12 & 18.1 & 19.4 & 1.3 \\
\hline Average & & \\
\hline${ }^{*} 1.5 \mathrm{~mm}$ & & & $1.35(-0.5-2.4)$ \\
\hline
\end{tabular}

Note: The average and maximum absolute errors of the selected points were $1.35 \mathrm{~mm}$ and $2.4 \mathrm{~mm}$, respectively. The total error range of absolute error was $\pm 1.5 \mathrm{~mm}$ according to the error range of the carved depth and the corresponding protruding depth. It was found that the outcomes met our system design errors. For all but one test point, the cutting depths were shallow than the depth of the protruding buttock. The more depth of cushion was carved, more absolute error occurred.

the points and reference plane on the buttock, we got the protruding depth according the pre-labeled landmarks. We designed the error range of the carved depth of the foam and the corresponding protruding depth of the buttock as $\pm 0.5 \mathrm{~mm}$ and $\pm 1 \mathrm{~mm}$, respectively. Table 2 presents the carved depth and the protruding depth (their corresponding buttock shape with sitting-posture) and their differences.

\subsection{Pressure-relief performance validation of the custom contoured cushion}

The subjective and objective evaluations were applied to the CCC and FC for the SCI and healthy participants. Subjective evaluations were obtained by questionnaire surveys. With regard to objective evaluations, the paired-sample $t$ test was used to analyze the two sets of objective data for all participants with FC and CCC, respectively.

Subjective evaluation: The LS, APS, and CD were applied to the questionnaire survey. There were ten scales in the questionnaire, with evaluation score ranging from one to ten. Higher scores indicate better performance of the cushion. Figure 2 shows the subjective evaluation of CCC and FC with healthy and SCI participants, respectively. The performances of the CCC had significant differences from flat cushion for the healthy and SCI patients $(\mathrm{p}<0.01)$. It can be concluded that the CCC improved LS, APS, and CD for both groups of study participants.

Objective evaluation: We evaluated the effect of CCC to uniform pressure distribution and decrease magnitude pressure interface for all subjects by analyzing raw pressure data. Mean value and standard deviation of MP, AP, APG, and BC with $\mathrm{CCC}$ and FC from all participants were described in Figure 3. The units of $\mathrm{APG}$ is in $\mathrm{mmHg} / \mathrm{cm}$ while $\mathrm{BC}$ is a dimensionless parameter. There were a significant 
differences for MP and AP with subjects seated on CCC and FC $(\mathrm{p}<0.01)$. The APG and BC for CCC were also significantly less than those of $F C(p<0.05)$.

Subjective evaluation of the healthy

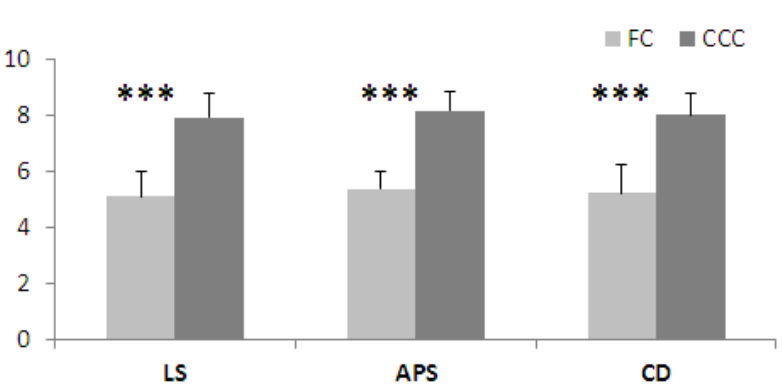

Subjective evaluation of $\mathrm{SCl}$

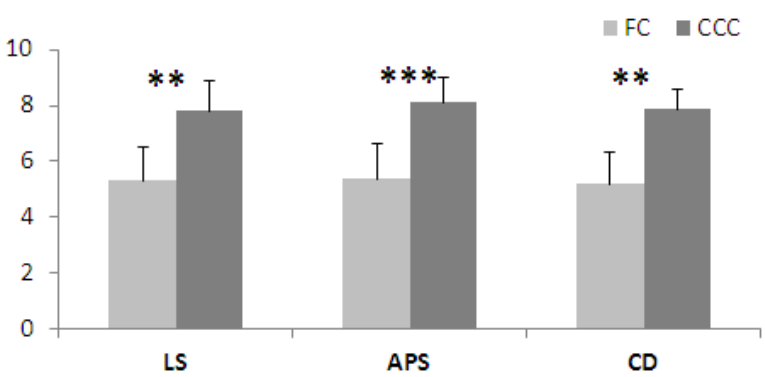

Fig. 2. The subjective evaluation parameters of the healthy and SCI. "***» $(p<0.005)$ and "***" $(p<0.01)$ mean that subjective evaluation of corresponding groups with $\mathrm{CCC}$ are significantly different from those of FC.
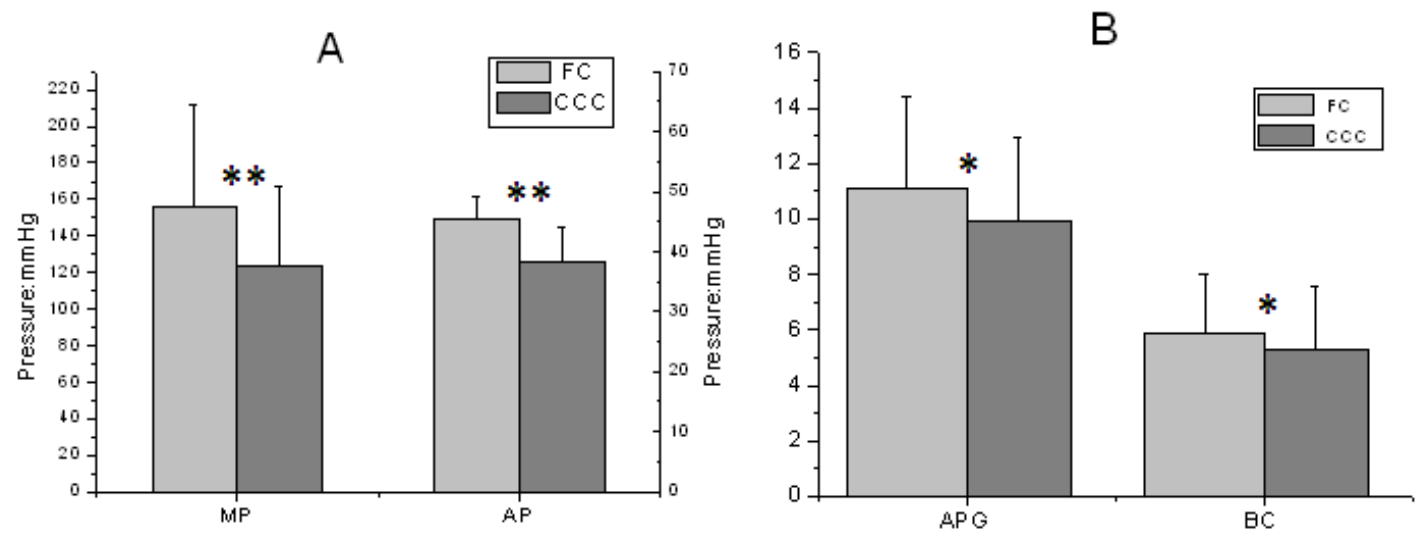

Fig. 3. The mean and SD of pressure distribution parameters. (A) gave the mean and SD (standard deviation) of the average pressure (AP) and maximum pressure (MP); (B) displayed the mean and SD of average pressure gradient (APG) and balance coefficient (BC).

\section{Discussion}

In the present study, a new method to manufacture seat cushion was developed. Our method employed interface pressure measurements representing the buttocks and upper-thigh topology to machining the cushion directly. We brought forward a new optimized algorithm of converting pressure distribution to the cutting depth according the load-deflection characteristics of the cushion foam. This algorithm normalized the peak carving depth and avoided the deeper cutting depth resulting in the CCC "bottoming out" under the ischial tuberosities, a high risk factor for developing pressure ulcers. 
The shape of the custom-contoured cushion was validated by comparing the custom-contoured cushion and its corresponding buttock shape in a seated posture. Since the carved depth of the foam and the corresponding protruding depth of the buttock were difficult to measure accurately, they were considered accurate to $\pm 0.5 \mathrm{~mm}$ and $\pm 1 \mathrm{~mm}$, respectively. Consequently, their percentage range of errors were $1-2.5 \%$ and $2 \sim 5 \%$ individually according to the measured values. The results demonstrated that more cutting depth resulted in more absolute error. This is because increasing depth resulted from the higher interface pressure which combined with the added deflection of soft tissue under buttock while the people sitting on the cushion, especially under the ischial tuberosities. The deflection of soft tissue increased the absolute error of the contoured foam cushion and its corresponding buttock shape. Although there were a few errors higher than our design, the magnitude of error was small enough such that force distributions were unaltered.

We researched the performances of custom-contoured cushions for different subjects. Paired-sample $\mathrm{t}$ test was performed on interface pressure with the FC and CCC for the healthy and SCI participants, respectively. The outcomes showed that the $\mathrm{CCC}$ redistributed and decreased the interface pressure between the buttocks and the cushion. In contrast with FC, the CCC could produce lower MP, AP, APG, and BC significantly. There results coincided with the previous study [14,18]. Since higher pressure parameters were associated with higher risk occurrences of pressure sores [25], the CCC would prevent pressure sores in high-risk individuals.

Nevertheless, three SCI subjects with hemiplegia raised MP and AP significantly. We deduce that these subjects may have difficulties in keeping a balanced pressure distribution on their bilateral buttocks when seated in a wheelchair, while fabricating the custom cushion, the higher pressure is, the more cutting depth is. Consequently, it would make force on bilateral buttocks more different, and that was the reason why the $\mathrm{CCC}$ of three hemiplegia participants could not relieve the interface pressure parameters. Therefore, the CCC is not suitable for patients with hemiplegia.

Subjective comfort rating depends on interface pressure distribution of buttocks, sitting position, measurement time, new situation adaptation and the duration of preceding experiments. To avoid the above impact factors, the subjective evaluations were given after maintaining standard sitting posture for thirty minutes. Since the CCC matched the buttocks shape better and provided more support than flat foam, this is why the CCC brought more stability, comfort, and alleviated the fatigue of partial muscular tissue.

Besides normal pressure, shear pressure, friction, and moisture also are the main causative factors to give rise to pressure ulcers [7]. In this study, we introduced special envelopment materials to release the shear force, friction and moisture. The envelopments have the characteristics of air permeability, moisture resistance, resilience, and damping, which makes the custom-contoured cushion provide the shear force and friction release and subjective comfortability.

\section{Conclusion}

This study proposed a new efficient method to design and manufacture custom-contoured cushion by converting the interface pressure under buttocks into the carving depth of cushions. The specialized system provided an effective, inexpensive and easy-manufacturing way of fabricating custom contoured foam cushion. Test findings demonstrated that the new CCC increased subjective comfort and stability and decreased interface pressures under the buttocks. This CCC would be of great benefit to patients, the elderly and prolonged sitting healthy subjects. 


\section{Acknowledgement}

The present study was supported by Postdoctoral Science Foundation of China, National Support Projects (No. 2012BAI33B01) and the Ministry of Education Science and Technology Innovation Program Development Fund (No. 708080). The researchers are particularly grateful to Xi'an Center Hospital and Xijing Hospital, Fourth Military Medical University, for their on the clinical experiments

\section{References}

[1] N. Reddy, T. Krouskop and P. Newell, Biomechanics of a lymphatic vessel, Journal of Vascular Research 12 (1975), 261-278.

[2] National Pressure Ulcer Advisory Panel, European pressure ulcer advisory panel \& national pressure ulcer advisory, prevention and treatment of pressure ulcers: Quick reference guide, Washington, 2009.

[3] D.M. Brienza et al., A manufacturing system for contoured foam cushions, Journal of Rehabilitation Research and Development 29 (1992), 32-40.

[4] R. Crenshaw and L. Vistnes, A decade of pressure sore research: 1977-1987, Journal of Rehabilitation Research and Development 26 (1989), 63-74.

[5] J. Reuler and T. Cooney, The pressure sore: Pathophysiology and principles of management, Annals of Internal Medicine 94 (1981), 661-666.

[6] R. Aissaoui et al., Analysis of pressure distribution at the body-seat interface in able-bodied and paraplegic subjects using a deformable active contour algorithm, Medical Engineering \& Physics 23 (2001), 359-367.

[7] D. Smith, Pressure ulcers in the nursing home, Annals of Internal Medicine 123 (1995), 433-442.

[8] G.M. Yarkony, Pressure ulcers-a review, Archives of Physical Medicine and Rehabilitation 75 (1994), $908-917$.

[9] J. Wang, Z. Ying et al., Rehabilitation Need of Persons with Disabilities in China: Analysis and Strategies for Deveploment, Huaxia Publishing House, Beijing, 2008.

[10] C. Gonzalez et al., Experimental seat for the study of the effects of random pneumatic stimulation for the prevention of pressure ulcers, The 2nd International Conference on Electrical and Electronics Engineering, 2005, 182-184.

[11] J.H. Hong et al., Development of an air seat cushion orthosis, Proceedings of the Second Joint Engineering in Medicine and Biology Society/Biomedical Engineering Society Conference, Houston, 2002, 2412-2413.

[12] A. Levy, K. Kopplin and A. Gefen, An air-cell-based cushion for pressure ulcer protection remarkably reduces tissue stresses in the seated buttocks with respect to foams: Finite element studies, Journal of Tissue Viability 23 (2014), 1323.

[13] Y, Yang and J. Wang, Design of an air-alternating anti-decubitus wheelchair seating system, 2009 IEEE Biomedical Circuits and Systems Conference (BioCAS), 2009, 261-264.

[14] D. Brienza, C. Lin and P. Karg, A method for custom-contoured cushion design using interface pressure measurements. IEEE Transactions on Rehabilitation Engineering 7 (1999), 99-108.

[15] D. Brienza, P. Karg and C. Brubaker, Seat cushion design for elderly wheelchair users based on minimization of soft tissue deformation using stiffness and pressure measurements, IEEE Transactions on Rehabilitation Engineering 4 (2002), 320-327.

[16] W.W. Chow and E.I. Odell, Deformation and stresses in soft body tissue of a sitting person, Journal of Biomechanical Engineering-Transactions of the Asme 100 (1978), 79-87.

[17] S.J. Li et al., A custom-contoured cushion for preventing pressure ulcers, Proceedings of the 2009 2nd International Conference on Biomedical Engineering and Informatics 1-4 (2009), 1074-1077.

[18] S. Sprigle, K. Chung and C. Brubaker, Reduction of sitting pressures with custom contoured cushions, Journal of Rehabilitation Research and Development 27 (1990), 135-140.

[19] C. Yang and Y. Hsu, Development of a rapid prototyping system for custom contoured foam cushion using the pressure mapping method, Biomedical Engineering: Applications, Basis and Communications 21 (2009), 9-16.

[20] D. Apatsidis, S. Solomonidis and S. Michael, Pressure distribution at the seating interface of custom-molded wheelchair seats: Effect of various materials, Archives of Physical Medicine and Rehabilitation 83 (2002), 1151-1156.

[21] W.W. Chow, Mechanical properties of gels and other materials with respect to their use in pads transmitting forces to the human body, Ph.D. Dissertation, The University of Michigan, 1974.

[22] F.P.D. Silva et al., Design and milling manufacture of polyurethane custom contoured cushions for wheelchair users, The Australasian Medical Journal 4 (2011), 500-506. 
[23] Y.C. Wang and R. Lakes, Analytical parametric analysis of the contact problem of human buttocks and negative poisson's ratio foam cushions, International Journal of Solids and Structures 39 (2002), 4825-4838.

[24] Y. Tanimoto et al., Pressure measurement of air cushions for SCI patients, IEEE Transactions on Instrumentation and Measurement 49 (2002), 666-670.

[25] D.M. Brienza et al., The relationship between pressure ulcer incidence and buttock-seat cushion interface pressure in atrisk elderly wheelchair users, Archives of Physical Medicine and Rehabilitation 82 (2001), 529-533. 